\title{
Output-Only Estimation of Amplitude Dependent Friction-Induced Damping
}

\author{
Vesterholm, Karsten K.; Friis, Tobias; Katsanos, Evangelos; Brincker, Rune; Brandt, Anders
}

Published in:

Dynamics of Civil Structures

Link to article, DOI:

10.1007/978-3-030-12115-0_3

Publication date:

2019

Document Version

Peer reviewed version

Link back to DTU Orbit

Citation (APA):

Vesterholm, K. K., Friis, T., Katsanos, E., Brincker, R., \& Brandt, A. (2019). Output-Only Estimation of Amplitude Dependent Friction-Induced Damping. In Dynamics of Civil Structures: Proceedings of the 37th IMAC, A Conference and Exposition on Structural Dynamics 2019 (Vol. 2, pp. 17-25). Springer. Conference Proceedings of the Society for Experimental Mechanics Series https://doi.org/10.1007/978-3-030-12115-0_3

\section{General rights}

Copyright and moral rights for the publications made accessible in the public portal are retained by the authors and/or other copyright owners and it is a condition of accessing publications that users recognise and abide by the legal requirements associated with these rights.

- Users may download and print one copy of any publication from the public portal for the purpose of private study or research.

- You may not further distribute the material or use it for any profit-making activity or commercial gain

- You may freely distribute the URL identifying the publication in the public portal 


\title{
Output-Only Estimation of Amplitude Dependent Friction-Induced Damping
}

\author{
Karsten K. Vesterholm ${ }^{1}$, Tobias Friis ${ }^{2}$, Evangelos Katsanos ${ }^{2}$, Rune Brincker ${ }^{2}$, Anders Brandt ${ }^{1}$ \\ ${ }^{1}$ University of Southern Demark, Department of Technology and Innovation \\ Campusvej 55, DK-5230 Odense M, Denmark \\ ${ }^{2}$ Technical University of Denmark, Danish Hydrocarbon Research and Technology Centre \\ Anker Engelunds Vej 1, DK-2800 Kgs. Lyngby, Denmark
}

\begin{abstract}
Identification of modal parameters, when a structure is under operational conditions is termed Operational Modal Analysis (OMA). Current OMA techniques are based on the assumption of linear time-invariant systems, and thus have limited applicability when applied to structures known to violate these assumptions. The present study investigates how the Random Decrement (RD) technique can improve robustness of OMA methods when friction-induced nonlinear damping is present in a system. This is done by estimating the amplitude dependent damping. A friction mechanism is introduced in a model of a structure, and by applying the $\mathrm{RD}$ technique at different amplitudes of simulated responses, RD signatures are produced, that represent the system vibrating with these amplitude levels. This allows the modal parameters to be estimated based on RD signatures computed with each amplitude level, using time domain parameter estimation methods, and the amplitude dependency of the damping is identified.
\end{abstract}

Keywords: random decrement, random vibrations, friction-induced nonlinear damping, operational modal analysis, identification of nonlinearity.

\section{INTRODUCTION}

The Random Decrement (RD) technique was invented in the late 1960's and the early 1970's by H. A. Cole [1-3], as a way to extract the 'signature' of a vibration signal, while the signal was being measured. It was invented to detect when damage in a vibrating structure occurred. The idea was, that damage was difficult to detect from observing the raw vibration signal, but easy to see in the signature. The RD technique is now an established output-only method for evaluating modal parameters of structures under random force inputs. It is possible to apply the RD technique, with a certain triggering condition, at multiple amplitude levels in the same response signal, and producing RD signatures that represent the system vibrating at these amplitude levels. The RD signatures can be treated as correlation functions, and amplitude specific modal parameters of the system can be estimated using time domain parameter estimation methods. When the modal parameters, representing different amplitude levels, are estimated, it reveals amplitude dependent nonlinearities in the system. The system investigated here has the stick-slip friction nonlinearity. The purpose of this study is to propose a new method of estimating the amplitude dependency of the damping caused by this type of nonlinearity, using only the measured response of the system. Amplitude dependent nonlinearities are present in many systems, and can cause difficulties, and erroneous results, since the current OMA methods are designed for linear systems [4].

The idea of applying the RD technique at multiple amplitude levels in one response signal was described by A. P. Jeary in 1992 [5], where it successfully uncovered the amplitude dependent damping in tall buildings. The damping was estimated by fitting a decay curve to the envelope of the auto RD signatures.

The local extremum (LE) triggering condition was first presented by Y. Tamura et al. in 1996 [6], as a method of evaluating amplitude dependent frequency and damping in buildings. The triggering condition was derived for a single-degree-of-freedom 
(SDOF) system, and experimental investigations of the amplitude dependent damping and frequency of 3 different towers was performed. In their methodology, the first step was to bandpass the response signals around the fundamental frequency with a narrow bandpass filter, to eliminate unwanted components. This analysis step can lead to erroneous results if the investigated system has closely spaced modes around the fundamental frequency. It is very plausible that this error is present in their results, given that two of the three towers are symmetric, and the third is symmetric to some extent, and are therefore highly likely to have closely spaced modes. Furthermore, the frequency and damping was estimated using the logarithmic decrement technique, where the peak values from the first two periods in the RD signature were used.

A resent study in 2018 by A. Bajrić et al. [7] investigated the output-only estimation of the system parameters in an SDOF system with hysteretic damping, using Covariance Driven Stochastic Subspace Identification (COV-SSI) and the linearization of a Bouc-Wen hysteresis model. The proposed method identified the system parameters robustly, but the method requires that the mass is known. Hysteresis damping and stick-slip friction have similar properties, where hysteresis could be considered a more versatile description of the physics in an actual damper [8].

The methods proposed in the present study are meant to improve robustness in Operational Modal Analysis (OMA), when applied to nonlinear systems. The numerical system being investigated consists of an underlying linear system, with a localized stick-slip friction present, and is therefore a nonlinear system. This type of nonlinearity is present in many places, for instance in the bridge bearings, connecting two off-shore platforms. One of the great challenges of this nonlinearity is that it depends not only on the present state and input of the system, but also on the previous state of the system.

To the authors' knowledge, no one has estimated the modal parameters of a numerical multiple-degree-of-freedom (MDOF) system with a localized stick-slip friction with the purpose of identifying the amplitude dependent damping as a function of the response, using the RD technique. The purpose of this study is therefore to investigate a novel approach of performing a $\mathrm{RD}$ analysis together with a time domain modal parameter estimation, that can estimate the amplitude dependent damping as function of the response. Matlab R2018a is used for simulation, analysis and visualization of results.

\section{THEORY}

This section describes the techniques and methods used during this study.

The present study applies the RD technique at multiple amplitude levels of simulated responses, and calculates the RD signatures using the LE triggering condition presented by Y. Tamura et al. [6], but the signal processing consisting of a bandpass filtering will be omitted to avoid the issues described above. The damping is estimated using a time domain parameter estimation method, where the auto- and cross RD signatures forms the equivalent of a correlation function matrix. With this method, many values in the RD signatures are used in the estimation, compared to the logarithmic decrement method used in [5, 6], where only the peak values from the first couple of periods were used. This means the damping estimates in the present study will have a higher statistical certainty, since it is based on more information. Also, since the RD signatures describe nonlinear vibrations, it cannot be assumed that the auto signature behaves entirely like an exponential decay, which is the assumption when using logarithmic decrement [8]. The limit of the approach proposed by A. Bajrić et al. is the assumption of a known mass. This assumption is not necessary for the approach proposed in the present study, which can result in an easier implementation. There is however, a trade-off between the two approaches, in the fact that Bajić et al. estimated the mechanical parameters, and it is therefore possible to reconstruct the system from the estimated parameters. The present study estimates modal parameters without scaling, where it is not possible to reconstruct the system, without additional information.

For a linear system, these two assumptions apply. One: the modal parameters do not change when estimating them with RD signatures calculated from different amplitude levels in the response signal [9]. Two: the modal parameters do not change when the amplitude of the zero mean Gaussian force signal exciting the system, is changed. The second assumption is based on the principle of superposition. These assumptions do not apply for the nonlinear system investigated in the present study. The $\mathrm{RD}$ analysis is used to describe the amplitude dependent damping of the system. For this to be successful, the case where the system is excited by one force level and analyzed at a specific response level, the damping estimate must be exactly the same as for a case where the system is excited by a different force level, while RD is applied at the same specific response level. This means that the modal parameters are found to be the same for a specific response level, regardless of the force level. 


\subsection{Random Decrement}

The RD signature is calculated as the average of $N$ segments extracted from a discrete stochastic process. The segments used in the averaging are found by identifying certain points, called triggering points, in the signal, that satisfy some condition, known as the triggering condition (TC). There are two types of RD signatures, the auto signature, where the TC is applied to, and the segments are extracted from the same signal. The cross signature is where the TC is applied to one signal, and the segments are extracted from another signal.

The cross RD signature is calculated by

$$
\hat{D}_{X, Y}(\tau)=\frac{1}{N} \sum_{i=1}^{N} x\left(t_{i}+\tau\right) \mid T_{y\left(t_{i}\right)}
$$

where $x$ is the signal, that $N$ segments are extracted from, and $T_{y\left(t_{i}\right)}$ is the triggering condition applied to signal $y$, identifying $N$ triggering points $t_{i}$. For the auto RD signature, $x$ and $y$ denote the same signal. The number of lags in the RD signature is controlled by the parameter $M$.

The triggering condition used in this study is the LE, where a triggering point is identified to be the local peak value, within a band of the response. This triggering condition was first proposed by Y. Tamura et al. to investigate amplitude dependent damping and frequency, where it was described as RD technique, ranked by peak amplitude. The TC was described more comprehensively by J. Asmussen [9], where the name LE was adopted, and formally defined as

$$
T_{y(t)}^{L E}=\left\{a_{1} \leq y(t)<a_{2}, \dot{y}(t)=0\right\}
$$

where $a_{1}$ and $a_{2}$ determine the band in the response, in which to find a peak, which occurs when $\dot{y}(t)=0$.

The analysis is performed on both the positive and negative parts of the response signal. Doing so, approximately doubles the number of triggering points, which helps limit the random error in the RD signature.

For the further analysis, RD signatures can be treated as correlation functions, and form the equivalent of a correlation function matrix, as done in $[4,9]$, and be used in the time domain modal parameter estimation.

\subsection{Multiple Reference Ibrahim Time Domain}

The time domain modal parameter estimation method used in this study is the Multiple Reference Ibrahim Time Domain (MITD) method.

The Ibrahim Time Domain (ITD) method, first published by S. R. Ibrahim et al. in 1977 [10], was developed to estimate modal parameters from free decays of linear systems, and is associated with the RD technique. ITD was extended to multiple references as described by R. J. Allemang et al. [11]. MITD is very similar to COV-SSI, the identification method used in [7]. The implementation of MITD is done in matlab with the ABRAVIBE toolbox [12].

\subsection{Equivalent linear damping}

A simplified approach to calculating the relative damping of a mode affected by a Coulomb type friction damping is to consider one period of the harmonic response of an SDOF system at resonance, i.e., a modal period, at a specific amplitude. Equalizing the dissipated energy of a combined linear and friction damped system with the dissipated energy of a linear system, the equivalent linear, viscous damping at a specific amplitude for a mode oscillating at its natural frequency becomes [13]:

$$
\zeta_{e q(n)}=\frac{1}{4 \pi} \frac{E_{d(n)}}{E_{t(n)}}=\frac{\pi \omega_{n} c_{n} Q_{n}+4 F d(n)}{2 \pi k_{n} Q_{n}}
$$

where $E_{d(n)}$ and $E_{t(n)}$ are the modal dissipated and total energy, respectively, and $\omega_{n}, k_{n}, c_{n}, Q_{n}, F d(n)$ are the $n$th modal components of the natural angular frequency, stiffness, viscous damping, displacement amplitude and friction force, respectively.

The equivalent linear damping is calculated to obtain a reference, with which to compare the results from the RD analysis. 


\section{NUMERICAL CASE STUDY}

\subsection{System investigated}

The system investigated in the present study is a numerical model, presented in Figure 1a, where the first 6 modes of the system are shown in Figure 1b. It is a T-shaped structure, that is clamped in its base, and has otherwise free boundary conditions, and is a 10 DOF system reduced from 156 DOFs [14]. The linear damping in the structure is implemented as modal damping, such that all modes have $1 \%$ relative damping. A stick-slip friction is positioned at DOF03, making the structure nonlinear.

The stick-slip friction describes a situation where two surfaces are in contact, for instance when a bridge is resting on a bridge bearing. There is friction between the two surfaces, meaning that when the bridge has a force acting on it, the resulting force has to overcome the friction, in order to move the bridge relative to the bearing. When the resulting force is not large enough to move the bridge, the system is in the 'stick' phase, and when the force overcomes the friction and moves the bridge, it is now in the 'slip' phase. In the slip phase, the friction force dissipates energy, meaning it acts as a damper. In the stick phase, the friction acts as a spring, which can have any stiffness, depending on the situation. This spring stiffness can describe different situations, and in this study, a very high stiffness is used together with a relatively low friction force compared to the external excitation, to maintain the mode shapes, and eigenfrequencies as in the linear case. This is done, since the purpose of this study is only to investigate an amplitude dependent damping alone.

For the T-structure, the stick-slip friction is implemented as Jenkins element going from DOF03 to ground. It is described by the parameters $F d$, the friction force, and $k d$, the spring stiffness. In this study the stick-slip parameters $F d=2.5 \mathrm{~N}$ and $k d=$ $10^{9} \frac{\mathrm{N}}{\mathrm{m}}$ are used. Moreover, with the chosen parameters of the friction element and the later defined magnitudes of the external excitation, the friction-induced damping is assumed to behave similarly to Coulomb friction. This means the friction force $F d$ is the one appearing in Equation 3. $k d$ is so high that we can use the simplified approach in Equation 3, and it does not appear in the Equation.

Since the stick-slip friction is acting in the direction of DOF03, it is expected that only modes that have motion in this direction are affected by the nonlinearity, meaning mode 2 and 5 in Figure $1 \mathrm{~b}$. It is expected that the only parameter of these modes that has an amplitude dependency is the relative damping.

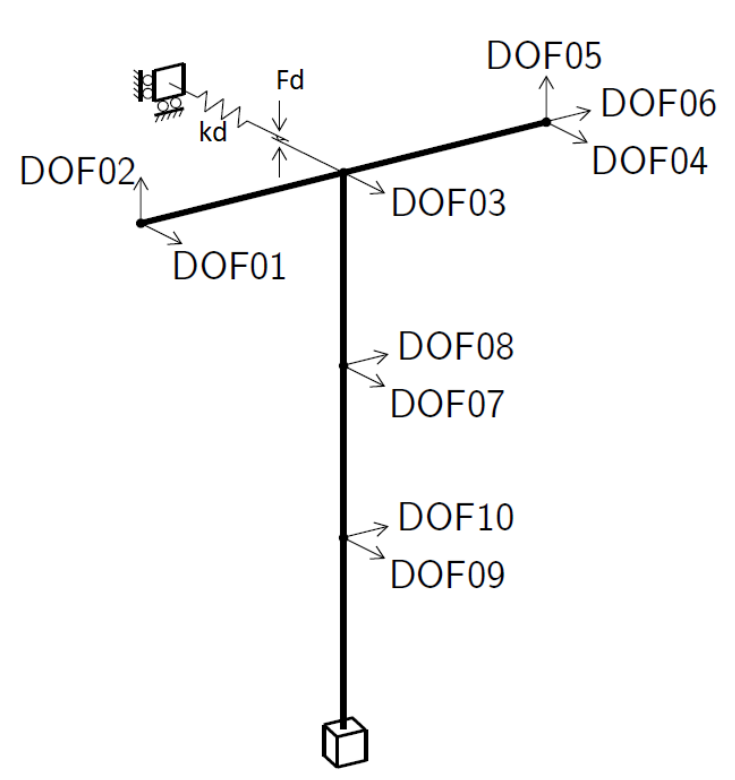

(a) 10 DOF model of T-structure, with stick-slip friction located at DOF03

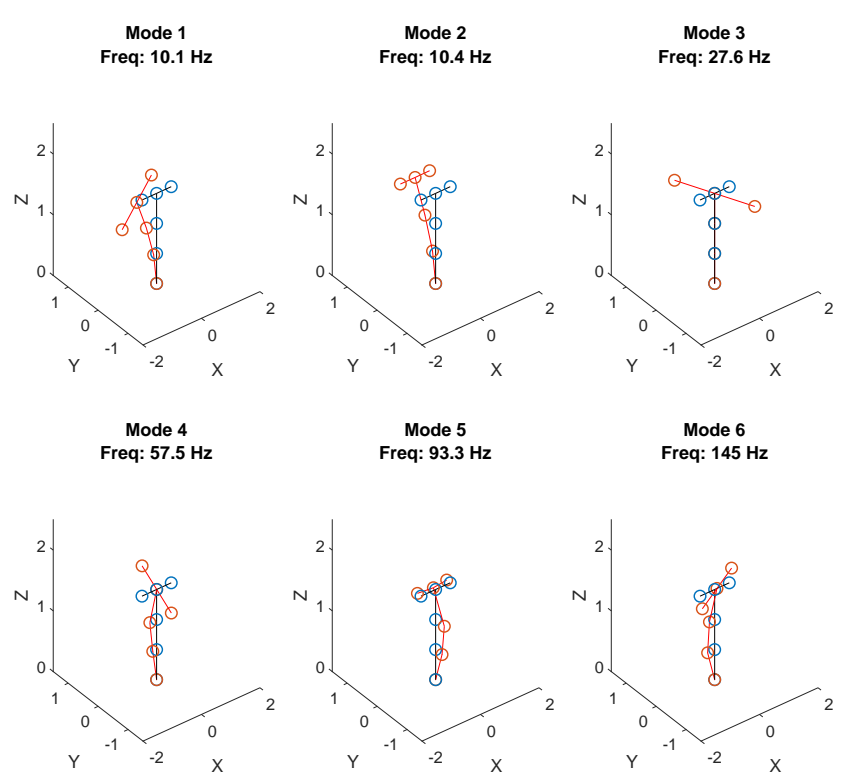

(b) First 6 mode shapes and frequencies

Fig. 1 Illustration of T-shaped structure. Notice that mode 1 and 2 are closely spaced, and that only mode 2 and 5 have in-phase motion in the y-direction 


\subsection{Analysis}

\subsubsection{SIMULATION PARAMETERS}

The numerical simulation is performed in matlab using a state space formulation as presented in [15], using a sampling frequency $f_{s}=2048 \mathrm{~Hz}$, and with a time history length of 30 minutes. A zero mean Gaussian signal is used as a force signal, and all DOFs are excited by an independent force signal, all with the same amplitude.

\subsubsection{RANDOM DECREMENT ANALYSIS PARAMETERS}

The number of lags in the RD signature, defined by the parameter $M$, must be large enough such that the number of lags in the RD signature passed to MITD can be adjusted during the investigations, and 512 is chosen. This leaves plenty of room for the analyst to tweak the number of lags in the RD signature passed to MITD.

Next, the triggering levels are defined. Firstly, the width of this triggering band, then at what response level it should be applied to is discussed. In Equation (2), $a_{1}$ and $a_{2}$ determine the band in the response, where the local peaks are found. The width of the triggering band is chosen to be constant for all the triggering levels. This ensures, that when the RD analysis is applied to different response levels, the amplitude is equally well defined for all triggering levels. This comes at a cost, in the form that the number of triggering points in each band is not equal, since the number of peaks in the signal, is not the same for all amplitudes. It has the consequence that the RD signatures calculated from different triggering levels, are not based on the same number of averages. It is assumed that this will not influence the results in this study, because of the long time histories. The width of the triggering band is defined as $3 \%$ of the signal range of the response from each DOF.

Recommendations for the optimal range of the response signal, in which to perform the RD analysis, are given by J. Asmussen [9]. These recommendations apply for a linear system, where the desire is to find the best level to trigger the signal to obtain the best RD signature. The recommendation is that triggering should not be performed below the standard deviation of the triggering signal $\sigma_{y}$, and not above $2 \sigma_{y}$.

When choosing triggering levels with the purpose of investigating the amplitude dependency, it is desired to have the largest range possible, and triggering levels between $1.2 \sigma_{y}$ and $2.5 \sigma_{y}$ are used in the present analysis. For higher triggering levels, the number of triggering points in the triggering band $\left[a_{1}, a_{2}\right]$ decrease to a point where the random error has a significant influence on the RD signatures. This random error propagates through the analysis and will increase the random error of the modal parameter estimates. For triggering levels below $1.2 \sigma_{y}$, a Monte Carlo simulation showed that the variance of damping estimates increase significantly, and few stable poles were obtained from MITD. This was discovered in the preliminary tests, where the parameters of the RD analysis were tweaked until enough stable poles were repeatedly obtained in the parameter estimation.

\subsubsection{MULTIPLE REFERENCE IBRAHIM TIME DOMAIN PARAMETERS}

Once the RD signatures are obtained, the modal parameters are estimated using the MITD method. There are no general recommendations for the number of lags that should be used in MITD, and reasoning behind the number chosen in the present study is discussed here.

The RD signature with LE, can be thought of as a method of investigating what happens, on average, a number of time lags after the system has experienced a local peak in the response, that has some specific amplitude. For a nonlinearity that is dependent on the systems' present and past states, it is not trivial or simple to explain, what happens a number of lags after a local peak in the response. The RD signature can therefore not be described as a linear model of the free decay, as the assumption for $\mathrm{RD}$ signatures is in MITD. Measures must be taken to mitigate this deviation from the linear assumption, in order to obtain good modal parameter estimates. An idea to solve this issue was proposed by R. Brincker et al. [16], where a nonlinear rocking system was investigated. The idea is to only use a number of lags covering the very first part of the RD signature, as an effort to minimize a bias in the RD signatures. This idea is adopted in the present study, and 110 lags from the RD signature are used in MITD. 110 lags cover a little more than half a period with the lowest frequency in the RD signature.

As a method of removing noise from RD signatures, in case any was present, the first 5 lags are omitted from all RD signatures, 
before passing them to the MITD method [17].

In summary, this means that the first 5 lags are skipped, and then the next 110 lags of the RD signatures are used in the MITD.

\subsubsection{MONTE CARLO SIMULATION}

To establish a baseline for the damping estimates $\hat{\zeta}$, a Monte Carlo simulation, using 50 runs, was performed with various excitation force levels. From each case with a different force level, the RD analysis was performed at 3 triggering levels, $1.2 \sigma_{y}$, $1.85 \sigma_{y}$, and $2.5 \sigma_{y}$. The specific triggering levels were different for each DOF, since the response level is different, and triggering levels used in the final Monte Carlo simulation was calculated from the triggering levels $1.2 \sigma_{y}, 1.85 \sigma_{y}$, and $2.5 \sigma_{y}$ for each force level, as an average of the triggering levels from 50 runs. This ensures that the same triggering level was used in the final Monte Carlo simulation. From the 50 runs, the standard deviation, and the mean of the damping estimates were calculated, and a region spanning from the mean \pm 3 standard deviation of the damping estimate $3 \sigma_{\hat{\zeta}}$ was formed. This was done for all the triggering levels in the different cases with different excitation force levels. The outcome of the Monte Carlo simulation will constitute the final results of this study, and be compared with the equivalent linear damping.

\subsubsection{EQUIVALENT LINEAR DAMPING}

The equivalent linear damping in Equation (3) is defined for a modal period, meaning the quantities needed must first be calculated, which is described in the following. The modal stiffness and modal damping, are found by pre- and post multiplying the stiffness and damping matrices from Section 3.1 with the real part of the estimated mode shape matrix $\Psi$, that has mode shape vectors as columns. To calculate the modal displacement values $Q_{n}$, a vector of linearly spaced displacement values going from 0.0005 to 0.01 is created and multiplied with mode shape 2 in DOF $3 \Psi_{3,2}$, meaning the third row in second column of $\Psi$. DOF 3 because it is chosen as the reference DOF, and mode 2 is used because it is the mode of interest regarding the results of the analysis.

\section{RESULTS AND DISCUSSION}

The results presented will have a main focus on mode 2 , since it is most affected by the nonlinearity.

When both a friction damper and a viscous damper are affecting a mode, which is the case for mode 2 , the relative damping $\zeta$ will decrease when the response level is increasing. The explanation is that the dissipation force in a friction damper is determined by a constant $F_{d}$, and the dissipation force for the linear term is the damping coefficient $c$ times velocity $\dot{y}$. For a low velocity, $F_{d}$ is the governing term regarding the dissipation of energy. This results in a high $\zeta$. When the response level increases, the linear dissipation force increases, while the friction damping force is constant. This results in lower $\zeta$, compared to when the response was lower.

For the various force levels investigated in the Monte Carlo simulation, the minimum number of triggering points in a response signal from the DOF's related to mode 2, was 930 in DOF03 when using a triggering level of $2.5 \sigma_{y}$. 930 triggering points is considered sufficient, but not excellent, to calculate an accurate RD signature. The number of triggering points changed from 1800 to 930 for the force levels $15.2 \mathrm{~N}$ and $55.6 \mathrm{~N}$, a phenomenon that does not occur in the purely linear case, and serves as an indicator that the system is nonlinear.

In Figure 2, the mean of the $\zeta$ estimates $\pm 3 \sigma_{\hat{\zeta}}$ for modes 1 and 2 from the Monte Carlo simulation are compared with the equivalent damping, given in Equation (3). The response from DOF03 is chosen to be the reference, and the triggering level on the $1^{\text {st }}$ axis is from DOF03. The Monte Carlo simulation, represented by Figure 2 shows that for mode $1, \hat{\zeta}$ is between 0.8 and $1.2 \%$. This is the expected result, and can be seen by two blue dashed lines enveloping the solid green line, representing the equivalent linear damping. This shows that the closely spaced modes 1 and 2 are successfully separated in the analysis. $\hat{\zeta}$ for mode 1 are as accurate as if the system had been linear.

$\hat{\zeta}$ of mode 2 , is found to be amplitude dependent, and inversely proportional to the triggering level. The two dashed black curves envelop the equivalent linear damping, the solid red curve. This means that the RD analysis with the MITD parameter estimation falls within $3 \sigma_{\hat{\zeta}}$ of the equivalent linear damping. This is considered a satisfactory result. However, there is still room 


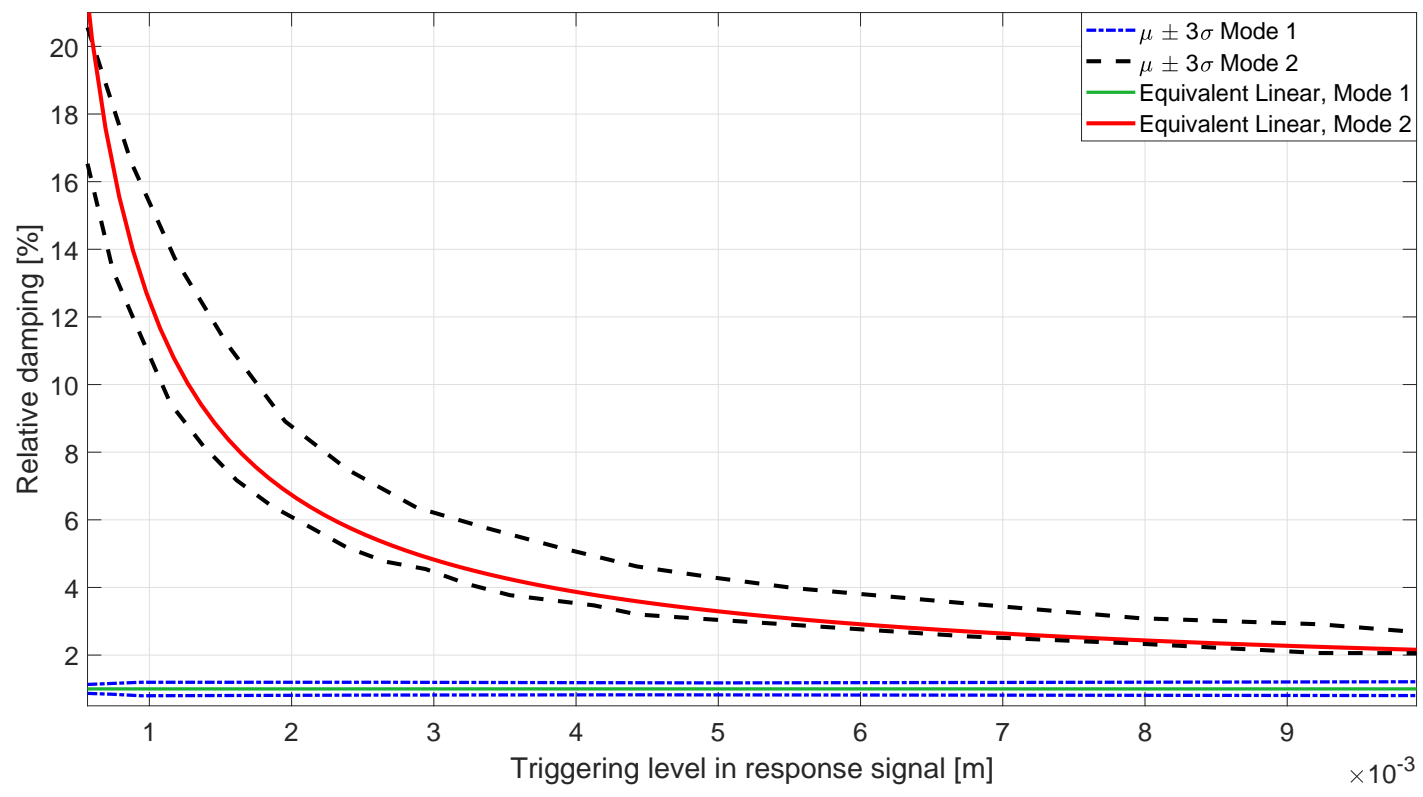

Fig. 2 Regions representing the damping estimates for modes 1 and 2 are illustrating the results from the Monte Carlo simulation. The region for mode 1 is between the two dot-dashed blue lines, representing $\mu_{\hat{\xi_{1}}} \pm 3 \sigma_{\hat{\zeta}_{1}}$. The region for mode 2 is between the two dashed black lines representing $\mu_{\hat{\zeta_{2}}} \pm 3 \sigma_{\hat{\zeta_{2}}}$. Inside the estimation regions for modes 1 and 2 , are the equivalent linear damping, the solid green and solid red lines respectively

for improvements. The width of the $\mu_{\hat{\zeta}} \pm 3 \sigma_{\hat{\zeta}}$ is very wide for some triggering levels, for instance at $10^{-3} \mathrm{~m}$. Here $\hat{\zeta}$ is estimated to be anywhere between $11 \%$ and $15.5 \%$, which is a very large margin.

The desire to have a RD analysis that estimates the same $\zeta$ for a particular triggering level, regardless of the force level applied to the system has not been satisfied in the present study. As the force level decreases, the damping estimates increase relative to triggering level. The phenomenon is most pronounced at the low triggering levels, where the damping is high. As the response level increases, the damping estimates for the different force levels start to meet each other, and eventually overlap when the damping estimate is around $4 \%$.

In Figure 2, $\mu_{\hat{\zeta}} \pm 3 \sigma_{\hat{\zeta}}$ for mode 2 has an increasing uncertainty as $\hat{\zeta}$ increases. Some of the increased uncertainty is caused by the nonlinearity, but not all of it. When a linear system has a large $\zeta$, the uncertainty of the estimate is also large. This is illustrated in Figure 3, where Monte Carlo simulations with 50 runs of linear systems with $\zeta$ of mode 2 ranging from $1 \%$ to $15 \%$, were performed. The analysis is otherwise exactly the same, as for the nonlinear system. The graph in Figure 3 , shows the mean \pm 3 standard deviations of the 25 estimates, against the different $\hat{\zeta}$ of mode 2 , and a trend is clear. The uncertainty of the damping estimate is increasing when the damping is increasing for linear systems. This increase in uncertainty should be taken into account, when interpreting the results in Figure 2.

Mode 5 is also affected by the stick-slip friction, but the influence is much more subtle. $\hat{\zeta}$ is found to be around $1.2 \%$, for the lowest triggering levels investigated, and drops quickly to $1 \%$ which is what the linear damping is.

A standard OMA analysis has been performed to compare with the results from the RD analysis. Everything is the same for the numerical model and Monte Carlo simulation. The differences between the analyses are that a correlation function matrix is calculated instead of a RD matrix, and the first 300 lags of the correlation functions are used in MITD. This is considered a standard OMA setting, and the results are presented in Table 1. It is not possible to show the results from the standard OMA analysis in the same plot as the RD analysis, because these damping estimates cannot be associated with a specific response level. This also means that the results are difficult to compare with the equivalent linear damping, meaning it is difficult to determine if the results are correct or not. The tendency of the standard OMA estimates is the same as for the RD analysis, that for a low force level, the relative damping is high, and it lowers when the force is increased. 


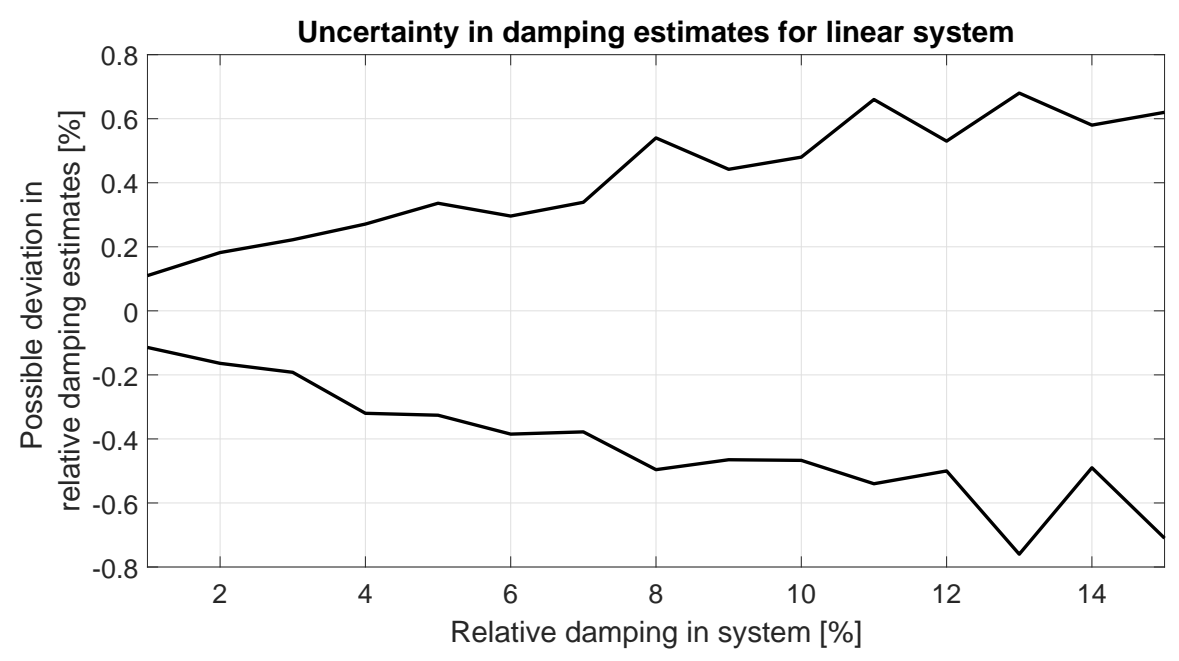

Fig. 3 From a Monte Carlo simulation with 25 runs, $3 \sigma_{\hat{\zeta}}$ is calculated and plotted against the linear damping in system with $\zeta$ ranging from $1 \%$ to $15 \%$, representing a possible deviation in the estimate

\begin{tabular}{|c|c|c|}
\hline RMS force level [N] & $\mu_{\hat{\zeta}} \%$ & $\mathbf{3} \sigma_{\hat{\zeta}} \%$ \\
\hline \hline 15.2 & 13.7 & 1.0264 \\
17.7 & 10.9 & 1.0734 \\
20.2 & 8.9 & 0.8195 \\
22.8 & 7.6 & 0.7512 \\
25.3 & 6.5 & 0.3815 \\
27.8 & 5.8 & 0.4716 \\
30.4 & 5.2 & 0.3859 \\
32.9 & 4.7 & 0.3171 \\
35.4 & 4.3 & 0.4214 \\
37.9 & 3.9 & 0.3134 \\
40.5 & 3.7 & 0.2935 \\
43.0 & 3.5 & 0.3641 \\
45.5 & 3.3 & 0.3223 \\
48.1 & 3.1 & 0.2949 \\
50.6 & 2.9 & 0.2167 \\
53.1 & 2.8 & 0.2197 \\
55.7 & 2.7 & 0.3018 \\
\hline
\end{tabular}

Table 1 Results from a Monte Carlo simulation of a standard OMA analysis using 50 runs. Excitation force in the left column, with corresponding mean and 3 standard deviations of damping estimate for mode 2 in middle and right column respectively

\section{CONCLUSION}

It can be concluded that the friction mechanism in DOF3 is causing the relative damping of modes 2 and 5 to exhibit amplitude dependent behavior.

The suggested method is successful in identifying that only modes 2 and 5 have amplitude dependent relative damping, even when modes 1 and 2 are closely spaced. The functional form of the damping estimates of mode 2 as a function of triggering level, is very similar to the equivalent linear damping. From this it can be concluded that the proposed approach to investigating the amplitude dependent damping of a system with a stick-slip friction mechanism is close to uncovering the parameters of the amplitude dependency, but further investigations are needed. Nothing about the system has to be known in order to perform this analysis, which means it could be used in structural dynamics output-only setting, to investigate the amplitude dependency of the relative damping. It can also be concluded that a standard OMA analysis cannot determine how the relative damping of mode 2 depends on the response amplitude with the same level of detail as the RD analysis is capable of. 


\section{ACKNOWLEDGEMENTS}

The authors acknowledge the funding received from Centre for Oil and Gas - DTU/Danish Hydrocarbon Research and Technology Centre (DHRTC).

\section{References}

[1] H. A. Cole. On-the-line analysis of random vibrations. In AIAA/ASME 9th Structural Dynamics Materials Conference, Palm Springs, USA, 1968.

[2] H. A. Cole. Failure Detection of a Space Shuttle Wing Flutter by Random Decrement. NASA, TMX-62,041, May, 1971.

[3] H. A. Cole. On-line failure detection and damping measurement of space structures by random decrement signatures. NASA CR-2205, 1973.

[4] Rune Brincker and Carlos Ventura. Introduction to operational modal analysis. John Wiley \& Sons, 2015.

[5] A. P. Jeary. Establishing non-linear damping characteristics of structures from non-stationary response time-histories. The Structural Engineer, 70:61-6, 1992.

[6] Yukio Tamura and Shin-ya Suganuma. Evaluation of amplitude-dependent damping and natural frequency of buildings during strong winds. Journal of wind engineering and industrial aerodynamics, 59(2):115-130, 1996.

[7] Anela Bajrić and Jan Høgsberg. Estimation of hysteretic damping of structures by stochastic subspace identification. Mechanical Systems and Signal Processing, 105:36-50, 2018.

[8] Singiresu S. Rao. Mechanical vibrations. Prentice Hall Upper Saddle River, 2011.

[9] John Christian Asmussen. Modal analysis based on the random decrement technique: application to civil engineering structures. PhD thesis, University of Aalborg, 1997.

[10] S. R. Ibraham and E. C. Mikulcik. A method for the direct identification of vibration parameter from the free responses. Shock and Vibration Bulletin, 47(4), 1977.

[11] Randall J. Allemang and David L. Brown. Experimental Modal Analysis and Dynamic Component Synthesis. Volume 3. Modal Parameter Estimation. Technical report, University of Cincinnati, Department of Mechanical and Industrial Engineering, 1987.

[12] Anders Brandt. Abravibe - a matlab toolbox for noise and vibration analysis and teaching, 2018.

[13] Anil K. Chopra. Dynamics of Structures. Pearson Education, 2012.

[14] J. O'Callahan. System equivalent reduction and expansion process. Proceedings of the 7th International Modal analysis conference, Society of Experimental Mechanics, pages 29-37, 1989.

[15] Lyan-Ywan Lu, Lap-Loi Chung, Lai-Yun Wu, and Ging-Long Lin. Dynamic analysis of structures with friction devices using discrete-time state-space formulation. Computers \& structures, 84(15-16):1049-1071, 2006.

[16] Rune Brincker, Milton Demosthenous, and George C. Manos. Estimation of the Coefficient of Restitution of Rocking Systems by the Random Decrement Technique. In Proceedings of the 12th International Modal Analysis Conference, 1994.

[17] Esben Orlowitz and Anders Brandt. Influence of noise in correlation function estimates for operational modal analysis. In Topics in Modal Analysis \& Testing, Volume 9, pages 55-64. Springer, 2019. 